\section{F. Koerner}

Received: 8 April 2005

Accepted: 11 April 2005

Published online: 28 June 2005

C) Springer-Verlag 2005

\author{
F. Koerner $(\bowtie)$ \\ University of Bern, \\ Bern, Switzerland \\ e-mail: fritz.koerner@bbox.ch
}

\title{
Vitreo-retinal surgery
}

The hardcover monograph entitled "Vitreo-retinal Surgery", edited by Bernd Kirchhof (Cologne) and David Wong (Liverpool), appeared as the 9th volume in the series 'Essentials in ophthalmology'. The reader might expect from the title a textbook with comprehensive instructions for surgical procedures for vitreo-retinal diseases. However, in the preface the authors point out that their aim is "to update our knowledge on the large body of experimental research performed to date on the most urgent problems of vitreoretinal diseases". Yet vitreo-retinal surgeons will find numerous implications of clinical relevance deducted from the outcome of actual basic research. Nevertheless, a title like "Modern Trends in Vitreoretinal Surgery" would perhaps have been more appropriate.

The 177-page book contains 11 chapters written by experienced researchers and clinicians. Each chapter is followed by an extensive list of alphabetically sorted references. A three-page subject index facilitates the search for specific topics. Each chapter is preceded by core messages, and subsections are followed by a 'Summary for the Clinician'. Most articles are illustrated with high-quality graphs and tables.

The reader will perhaps miss a chapter on currently available instrumentation and a description of standard procedures of vitreo-retinal surgery. The volume starts with an article on retinal implants by $P$. Walter. It deals mainly with technical solutions currently being investigated. The applicability of retinal implants in blind human patients needs to be awaited. A chapter of Antonia Joussen and B. Kirchhof summarizes the pathophysiology and actual pharmacological and surgical approaches against macular edema. Advances in the surgical removal of non-diabetic epimacular membranes and the internal limiting membrane (ILM) are the subject of an article of Silvia Bopp. An analysis of post-operative data illustrates the functional benefit of so-called pucker surgery. Pros and cons of the use of dyes are discussed. Because of the risk of postoperative cataract formation, the author recommends a combination of the vitrectomy with a cataract operation or clear lens extraction in all patients over 50 years of age. However, this message needs to be proven by prospective controlled studies. Two articles deal with some aspects of the surgical management of age-related macular degeneration (AMD). A critical review by J. Roider leaves the reader with substantial doubt about the effectiveness of 'transpupillary thermotherapy' (TTT). J.C. van Meurs describes a new approach beyond macular translocation. It focuses on the need of autologous translocation of a sheet of retinal pigment epithelial cells (RPE) from the mid-periphery to the diseased macular area. Preliminary results are reported to be promising although post-operative proliferative vitreoretinopathy (PVR) had to be treated in the majority of 18 patients. Present knowledge of the activation 
and differentiation of RPE cells under experimental and clinical conditions is presented in a chapter by C. Sheridan et al. A clinically oriented overview of the etiology and management of retinal giant tears is given in an article by M. Snead. Risk factors and various measures in preventing PVR are the subject of two chapters by C.H. Kon et al. and D.G. Charteris, respectively. Besides well-known biological and surgical risk factors, an interesting 'PVR score' based on multivariate statistics is cited the use of which needs to be proven in clinical praxis. Unfortunately, the formula of the score given in both chapters is not quite identical due to a misprint. The article on prevention of PVR fails to mention the potential effect of local and systemic corticosteroids that are apparently often used by surgeons on a 'non-evidence basis'. The background of the various effects and side effects of tamponade agents is explained in an article by D. Wong. The book closes with a chapter of T.H. Williamson on various aspects of vitreo-retinal surgery in inflammatory diseases and lymphoma.

The book is quite easy to read. It is written in perfect English. I would recommend this volume as complement reading for vitreo-retinal surgeons and researchers as well as for residents and fellows in ophthalmology. The book may also serve as a very useful searching tool for recent publications in the field. 